\title{
A GROWING ALGEBRA CONTAINING THE POLYNOMIAL RING
}

\author{
Seul Hee Choi
}

\section{Abstract}

There are various papers on finding all the derivations of a nonassociative algebra and an anti-symmetrized algebra (see [2], [3], [4], $[5],[6],[10],[13],[15],[16])$. We find all the derivations of the growing algebra $W N\left(e^{ \pm x_{1} x_{2} x_{3}}, 0,3\right)_{[1]}$ with the set of all right annihilators $T_{3}=$ $\left\{i d, \partial_{1}, \partial_{2}, \partial_{3}\right\}$ in the paper. The dimension of $\operatorname{Der}_{n o n}\left(W N\left(e^{ \pm x_{1} x_{2} x_{3}}, 0\right.\right.$, $\left.3)_{[1]}\right)$ of the algebra $W N\left(e^{ \pm x_{1} x_{2} x_{3}}, 0,3\right)_{[1]}$ is one and every derivation of the algebra $W N\left(e^{ \pm x_{1} x_{2} x_{3}}, 0,3\right)[1]$ is outer. We show that there is a class $\mathfrak{P}$ of purely outer algebras in this work.

\section{Preliminaries}

Let $\mathbb{N}$ be the set of all non-negative integers and $\mathbb{Z}$ be the set of all integers. Let $\mathbb{N}^{+}$be the set of all positive integers. Let $\mathbb{F}$ be a field of characteristic zero and $\mathbb{F}^{\bullet}$ the set of all non-zero elements in $\mathbb{F}$. Throughout the paper, we will assume that $e$ is not the element of the field $\mathbb{F}$. For $n, t \in \mathbb{N}$, throughout the paper, $m$ denotes a nonnegative integer such that $m \leq n+t$. For fixed integers, $i_{1}, \cdots, i_{m}$ and for given irreducible polynomials $f_{1}, \cdots, f_{m} \in \mathbb{F}\left[x_{1}, \cdots, x_{n+t}\right]$, define $\left[f_{1}^{i_{1}}, \cdots, f_{m}^{i_{m}}\right]$ as the set Poly P $_{m}=P_{m}=\left\{f_{1}^{i_{1}} \cdots f_{m}^{i_{m}}, f_{1}^{i_{1}} \cdots f_{m-1}^{i_{m-1}}, \cdots\right.$, $\left.f_{2}^{i_{2}} \cdots f_{m}^{i_{m}}, \cdots, f_{1}^{i_{1}}, \cdots, f_{m}^{i_{m}}\right\}$. For any subset $P$ of $P_{m}$, define the $\mathbb{F}$ algebra $\mathbb{F}\left[e^{ \pm[P]}, n, t\right]:=\mathbb{F}\left[e^{ \pm[P]}, x_{1}^{ \pm 1}, \cdots, x_{n}^{ \pm 1}, x_{n+1}, \cdots, x_{n+t}\right]$, which is

Received June 9, 2010. Accepted September 1, 2010.

Key words and phrases: purely outer, non-associative algebra, derivation. Mathematics Subject Classification: Primary 17B40, $17 B 56$. 
spanned by

$$
\begin{aligned}
& \mathbf{B}=\left\{e^{a_{1} f_{1}} \cdots e^{a_{r} f_{r}} x_{1}^{j_{1}} \cdots x_{n+t}^{j_{n+t}} \mid f_{1}, \cdots, f_{r} \in P, a_{1}, \cdots, a_{r} \in \mathbb{Z},\right. \\
& \left.j_{1}, \cdots, j_{n} \in \mathbb{Z}, j_{n+1}, \cdots, j_{n+t} \in \mathbb{N}\right\}
\end{aligned}
$$

We then denote $\partial_{h_{1}}^{k_{1}} \cdots \partial_{h_{r}}^{k_{r}}$ by the composition of the partial derivatives $\partial_{h_{1}}, \cdots, \partial_{h_{r}}$ on $\mathbb{F}\left[e^{ \pm[P]}, n, t\right]$ with appropriate exponents where $1 \leq$ $h_{1}, \cdots, h_{r} \leq n+t$ and $\partial_{h}^{0}, 1 \leq h \leq n+t$, denotes the identity map on $\mathbb{F}\left[e^{ \pm[P]}, n, t\right]$. For any $\alpha_{u} \in P \subset P_{m}$, let $\mathfrak{A}_{\alpha_{u}}$ be an additive subgroup of $\mathbb{F}$ such that $\mathfrak{A}_{\alpha_{u}}$ contains $\mathbb{Z}$. Consider now the (free) $\mathbb{F}$-vector space $N\left(e^{\mathfrak{A}_{P}}, n, t\right)_{k}$ (resp. $\left.N\left(e^{\mathfrak{A}_{P}}, n, t\right)_{k^{+}}\right)$whose basis is the set

$$
\mathbf{B}_{1}=\left\{e^{a_{1} f_{1}} \cdots e^{a_{r} f_{r}} x_{1}^{j_{1}} \cdots x_{n+t}^{j_{n+t}} \partial_{h_{1}}^{k_{1}} \cdots \partial_{h_{r}}^{k_{r}} \mid a_{1} \in \mathfrak{A}_{\alpha_{1}}, \cdots, a_{r} \in \mathfrak{A}_{\alpha_{r}},\right.
$$

(1) $f_{1}, \cdots, f_{r} \in P, h_{1}, \cdots, h_{r} \leq n+t, k_{1}+\cdots+k_{r} \leq k \in \mathbb{N}$

$\left.\left(\operatorname{resp} . \mathbb{N}^{+}\right)\right\}$

If we define the multiplication $*$ on $N\left(e^{\mathfrak{A}_{P}}, n, t\right)_{k}$ as follows:

$$
f \partial_{h_{1}}^{p_{1}} \cdots \partial_{h_{r}}^{p_{r}} * g \partial_{u_{1}}^{v_{1}} \cdots \partial_{u_{q}}^{v_{q}}=f\left(\partial_{h_{1}}^{p_{1}} \cdots \partial_{h_{r}}^{p_{r}}(g)\right) \partial_{u_{1}}^{v_{1}} \cdots \partial_{u_{q}}^{v_{q}}
$$

for any $f \partial_{h_{1}}^{p_{1}} \cdots \partial_{h_{r}}^{p_{r}}, g \partial_{u_{1}}^{v_{1}} \cdots \partial_{u_{q}}^{v_{q}} \in N\left(e^{\mathfrak{A}_{P}}, n, t\right)_{k}$, then we define the combinatorial non-associative algebra $W N\left(e^{\mathfrak{A}_{P}}, n, t\right)_{k}$ whose underlying vector space is $N\left(e^{\mathfrak{A}_{P}}, n, t\right)_{k}$ and whose multiplication is $*$ in (2) (see [1], [2], [5], [14] and [15]). The non-associative subalgebra $W N\left(e^{\mathfrak{A}_{P}}, n, t\right)_{<k>}$ of the algebra $W N\left(e^{\mathfrak{A}_{P}}, n, t\right)_{k}$ is generated by

(3) $\left\{f \partial_{h_{1}}^{k_{1}} \cdots \partial_{h_{r}}^{k_{r}} \mid f \in \mathbf{B}, 1 \leq h_{1}, \cdots, h_{r} \leq n+t, k_{1}+\cdots+k_{r}=k \in \mathbb{N}^{+}\right\}$.

The non-associative subalgebra $W N\left(e^{\mathfrak{A}_{P}}, n, t\right)_{[k]}$ of the algebra $W N\left(e_{P}^{\mathfrak{A}}\right.$, $n, t)_{k}$ is generated by

$$
\left\{f \partial_{h}^{k} \mid f \in \mathbf{B}, 1 \leq h \leq n+t\right\} .
$$

For an algebra $A$ and $l \in A$, an element $l_{1} \in A$ is a right (resp. left) identity of $l$, if $l * l_{1}=l$ (resp. $l_{1} * l=l$ ) holds. The set of all right identities of $W N\left(e^{\mathfrak{A}_{P}}, n, t\right)_{[1]}$ is $\left\{\sum_{1 \leq u \leq n+t} x_{u} \partial_{u}+\sum_{1 \leq u \leq n+t} c_{u} \partial_{u} \mid c_{u} \in \mathbb{F}\right\}$. There is no left identity of $W N\left(e^{\mathfrak{A} \mathfrak{A}_{P}}, n, t\right)_{k^{+}}$. The algebra $W N\left(e^{\mathfrak{A}_{P}}, n, t\right)_{k}$ has the left identity 1 . If $A$ is an associative $\mathbb{F}$-algebra, then the antisymmetrized algebra of $A$ is a Lie algebra relative to the commutator $[x, y]:=x y-y x$, (See [9]). For a general non-associative $\mathbb{F}$-algebra $N$ we define in the same way its antisymmetrized algebra $N^{-}$. In case $N^{-}$is a Lie algebra we shall say that $N$ is Lie admissible. For $S \subset N^{-}$, an element $l$ is ad-diagonal with respect to $S$ if for any $l_{1} \in S,\left[l, l_{1}\right]=c l_{1}$ for $c \in \mathbb{F}$. The algebra $W N\left(e^{\mathfrak{A}_{P}}, n, t\right)_{[1]}$ is Lie admissible (see [1], [7], [16], and [18]). 
Since the cardinality $|P|$ of $P$ is $2^{m}$, for all $\alpha \in P_{m}$, if $\mathfrak{A}_{\alpha}$ is $\mathbb{Z}$, then the algebra $W N\left(e^{\mathfrak{A}_{P_{m}}}, n, t\right)_{k}$ is $\mathbb{Z}^{2^{m}}$-graded as follows:

$$
W N\left(e^{\mathfrak{A}_{P_{m}}}, n, t\right)_{k}=\bigoplus_{\left(a_{1}, \cdots, a_{m^{2}}\right)} N_{\left(a_{1}, \cdots, a_{m^{2}}\right)}
$$

where $\left.N_{\left(a_{1}, \cdots, a_{2} m\right.}\right)$ is the vector subspace of $W N\left(e^{\mathfrak{A}_{P_{m}}}, n, t\right)_{k}$ spanned by

$$
\left\{e^{a_{1} f_{1}} \cdots e^{a_{r} f_{r}} x_{1}^{j_{1}} \cdots x_{n+t}^{j_{n+t}} \mid j_{1}, \cdots, j_{n} \in \mathbb{Z}, j_{n+1}, \cdots, j_{n+t} \in \mathbb{N}\right\} .
$$

This implies that $W N\left(e^{\mathfrak{A}_{P}}, n, t\right)_{k}$ and $W N\left(e^{\mathfrak{A}_{P}}, n, t\right)_{k^{+}}$are appropriate graded algebras as (5) (see [11]). Thus throughout the paper, the $(0, \cdots, 0)$-homogeneous component $N_{0}$ of $W N\left(e^{\mathfrak{A}_{P}}, n, t\right)_{k}$ is the subalgebra $W N(0, n, t)_{k}$ of $W N\left(e^{\mathfrak{A}_{P}}, n, t\right)_{k}$. For any standard basis element $e^{a_{1} f_{1}} \cdots e^{a_{r} f_{r}} x_{1}^{j_{1}} \cdots x_{n+t}^{j_{n+t}} \partial_{t_{1}}^{k_{1}} \cdots \partial_{t_{r}}^{k_{r}}$ of $W N\left(e^{\mathfrak{A}_{P m}}, n, t\right)_{k}$, define the homogeneous degree as follows:

$$
h d\left(e^{a_{1} f_{1}} \cdots e^{a_{r} f_{r}} x_{1}^{j_{1}} \cdots x_{n+t}^{j_{n+t}} \partial_{t_{1}}^{k_{1}} \cdots \partial_{t_{r}}^{k_{r}}\right)=\sum_{u=1}^{n+t}\left|j_{u}\right|
$$

where $\left|j_{u}\right|$ is the absolute value of $j_{u}$ for $1 \leq u \leq n+t$. For any element $l \in W N\left(e^{\mathfrak{A}_{P}}, n, t\right)_{k}$, define $h d(l)$ as the highest homogeneous degree of each monomial of $l$. Note that the set of all right annihilators of $W N\left(e^{\mathfrak{A}_{P}}, n, t\right)_{k}$ is the subalgebra $T_{n+t}$ of $W N\left(e^{\mathfrak{A}_{P}}, n, t\right)_{k}$ which is spanned by $\left\{\partial_{t_{1}}^{k_{1}} \cdots \partial_{t_{r}}^{k_{r}} \mid 1 \leq t_{1}, \cdots, t_{r} \leq n+t, k_{1}+\cdots+k_{r} \leq k \in \mathbb{N}\right\}$. For a given algebra $A, \operatorname{Out}(A)$ (resp. $\operatorname{Inn}(A))$ is the set of all the outer (resp. inner) derivations of $A$ and $\operatorname{Der}(A)$ is the set of all the derivations of $A$. An algebra $A$ is purely outer, if every derivation of $A$ is outer i.e., $\operatorname{Der}(A)=\operatorname{Out}(A)$.

3. Derivations of the non-associative algebra $W N\left(e^{ \pm x_{1} x_{2} x_{3}}, 0\right.$, $3)_{[1]}$

For this section, the set of all right annihilators $T_{3}$ of $W N\left(e^{ \pm x_{1} x_{2} x_{3}}, 0\right.$, $3)_{[1]}$ is spanned by $\left\{i d, \partial_{1}, \partial_{2}, \partial_{3}\right\}$. The algebra $W N\left(e^{ \pm x_{1} x_{2} x_{3}}, 0,3\right)_{[1]}$ contains the polynomial ring and it is simple (see [4]).

Note 1. For any basis elements $\partial_{u}, x_{1}^{i_{1}} x_{2}^{i_{2}} x_{3}^{i_{3}}, x_{1}^{i_{1}} x_{2}^{i_{2}} x_{3}^{i_{3}} \partial_{u}, e^{p x_{1} x_{2} x_{3}} x_{1}^{i_{1}} x_{2}^{i_{2}} x_{3}^{i_{3}}$, $e^{p x_{1} x_{2} x_{3}} x_{1}^{i_{1}} x_{2}^{i_{2}} x_{3}^{i_{3}} \partial_{u}, 1 \leq u \leq 3$, of $W N\left(e^{ \pm x_{1} x_{2} x_{3}}, 0,3\right)_{1}$, and for any $c \in$ 
$\mathbb{F}, p \in \mathbb{Z}$, if we define an $\mathbb{F}$-linear map $D_{c}$ from the algebra $W N\left(e^{ \pm x_{1} x_{2} x_{3}}, 0\right.$, $3)_{1}$ to itself as follows:

$$
\begin{aligned}
& D_{c}\left(\partial_{u}\right)=0, \\
& D_{c}\left(x_{1}^{i_{1}} x_{2}^{i_{2}} x_{3}^{i_{3}}\right)=0, \\
& D_{c}\left(x_{1}^{i_{1}} x_{2}^{i_{2}} x_{3}^{i_{3}} \partial_{u}\right)=0, \\
& D_{c}\left(e^{p x_{1} x_{2} x_{3}} x_{1}^{i_{1}} x_{2}^{i_{2}} x_{3}^{i_{3}}\right)=p c e^{p x_{1} x_{2} x_{3}} x_{1}^{i_{1}} x_{2}^{i_{2}} x_{3}^{i_{3}}, \\
& D_{c}\left(e^{p x_{1} x_{2} x_{3}} x_{1}^{i_{1}} x_{2}^{i_{2}} x_{3}^{i_{3}} \partial_{u}\right)=p c e^{p x_{1} x_{2} x_{3}} x_{1}^{i_{1}} x_{2}^{i_{2}} x_{3}^{i_{3}} \partial_{u},
\end{aligned}
$$

then the map $D_{c}$ can be linearly extended to a non-associative algebra derivation of

$W N\left(e^{ \pm x_{1} x_{2} x_{3}}, 0,3\right)_{[1]}$ where $1 \leq u \leq 3$ (see [6], [7] and [10]).

Lemma 3.1. For any derivation $D$ of $W N\left(e^{ \pm x_{1} x_{2} x_{3}}, 0,3\right)_{[1]}$ and for any basis elements $\partial_{u}, x_{1}^{i_{1}} x_{2}^{i_{2}} x_{3}^{i_{3}}, x_{1}^{i_{1}} x_{2}^{i_{2}} x_{3}^{i_{3}} \partial_{u}, 1 \leq u \leq 3$, of $W N\left(e^{ \pm x_{1} x_{2} x_{3}}\right.$, $0,3)_{[1]}$, we have that

$$
\begin{aligned}
& D\left(\partial_{u}\right)=0, \\
& D\left(x_{1}^{i} x_{2}^{j} x_{3}^{k}\right)=i c_{0,0,0,1} x_{1}^{i-1} x_{2}^{j} x_{3}^{k}+j d_{0,0,0,2} x_{1}^{i} x_{2}^{j-1} x_{3}^{k}+k r_{0,0,0,3} x_{1}^{i} x_{2}^{j} x_{3}^{k-1}, \\
& D\left(x_{1}^{i} x_{2}^{j} x_{3}^{k} \partial_{u}\right)=i c_{0,0,0,1} x_{1}^{i-1} x_{2}^{j} x_{3}^{k} \partial_{u}+j d_{0,0,0,2} x_{1}^{i} x_{2}^{j-1} x_{3}^{k} \partial_{u}+k r_{0,0,0,3} x_{1}^{i} \\
& x_{2}^{j} x_{3}^{k-1} \partial_{u}
\end{aligned}
$$

hold with appropriate coefficients where $1 \leq u \leq 3$.

Proof. Let $D$ be the derivation in the lemma. Since the algebra $W N\left(e^{ \pm x_{1} x_{2} x_{3}}, 0,3\right)_{[1]}$ is $\mathbb{Z}$-graded, $D\left(\partial_{1}\right)$ is the sum of terms in different homogeneous components of $W N\left(e^{ \pm x_{1} x_{2} x_{3}}, 0,3\right)_{[1]}$ in (5). Thus $D\left(\partial_{1}\right)$ can be written as follows:

$$
\begin{aligned}
D\left(\partial_{1}\right) & =\sum_{i, j, k \geq 0} \alpha_{i, j, k, 0} e^{p x_{1} x_{2} x_{3}} x_{1}^{i} x_{2}^{j} x_{3}^{k}+\sum_{i, j, k \geq 0} \alpha_{i, j, k, 1} e^{p x_{1} x_{2} x_{3}} x_{1}^{i} x_{2}^{j} x_{3}^{k} \partial_{1} \\
& +\sum_{i, j, k \geq 0} \alpha_{i, j, k, 2} e^{p x_{1} x_{2} x_{3}} x_{1}^{i} x_{2}^{j} x_{3}^{k} \partial_{2}+\sum_{i, j, k \geq 0} \alpha_{i, j, k, 3} e^{p x_{1} x_{2} x_{3}} x_{1}^{i} x_{2}^{j} x_{3}^{k} \partial_{3}
\end{aligned}
$$


with appropriate coefficients. Since $\partial_{1}$ centralizes itself, we have that $D\left(\partial_{1}\right)$ is in the right annihilator of $\partial_{1}$, i.e.,

$$
\begin{aligned}
\partial_{1} * D\left(\partial_{1}\right) & =\sum_{i, j, k \geq 0} p \alpha_{i, j, k, 0} e^{p x_{1} x_{2} x_{3}} x_{1}^{i} x_{2}^{j+1} x_{3}^{k+1} \\
& +\sum_{i \geq 1, j, k \geq 0} i \alpha_{i, j, k, 0} e^{p x_{1} x_{2} x_{3}} x_{1}^{i-1} x_{2}^{j} x_{3}^{k} \\
& +\sum_{i, j, k \geq 0} p \alpha_{i, j, k, 1} e^{p x_{1} x_{2} x_{3}} x_{1}^{i} x_{2}^{j+1} x_{3}^{k+1} \partial_{1} \\
& +\sum_{i \geq 1, j, k \geq 1} i \alpha_{i, j, k, 1} e^{p x_{1} x_{2} x_{3}} x_{1}^{i-1} x_{2}^{j} x_{3}^{k} \partial_{1} \\
& +\sum_{i, j, k \geq 0} p \alpha_{i, j, k, 2} e^{p x_{1} x_{2} x_{3}} x_{1}^{i} x_{2}^{j+1} x_{3}^{k+1} \partial_{2} \\
& +\sum_{i \geq 1, j, k \geq 0} i \alpha_{i, j, k, 2} e^{p x_{1} x_{2} x_{3}} x_{1}^{i-1} x_{2}^{j} x_{3}^{k} \partial_{2} \\
& +\sum_{i, j, k \geq 0} p \alpha_{i, j, k, 3} e^{p x_{1} x_{2} x_{3}} x_{1}^{i} x_{2}^{j+1} x_{3}^{k+1} \partial_{3} \\
& +\sum_{i \geq 1, j, k \geq 0} i \alpha_{i, j, k, 3} e^{p x_{1} x_{2} x_{3}} x_{1}^{i-1} x_{2}^{j} x_{3}^{k} \partial_{3} \\
& =0
\end{aligned}
$$

with appropriate coefficients. By (7), we have that $\alpha_{i, j, k, 0}, \alpha_{i, j, k, 1}, \alpha_{i, j, k, 2}$, and $\alpha_{i, j, k, 3}$, are zeros, $i, j, k \geq 0$. Thus $D\left(\partial_{1}\right)$ is zero. Similarly, we can prove that $D\left(\partial_{2}\right)$ and $D\left(\partial_{3}\right)$ are also zeros. By $D\left(\partial_{u} * x_{1}\right)=0,1 \leq u \leq 3$, we can prove that $D\left(x_{1}\right)=b_{0,0,0,0}+b_{0,0,0,1} \partial_{1}+b_{0,0,0,2} \partial_{2}+b_{0,0,0,3} \partial_{3}$. Similarly, since $\partial_{u}$ centralizes $x_{1} \partial_{1}$, we can also prove that

$$
D\left(x_{1} \partial_{1}\right)=c_{0,0,0,0}+c_{0,0,0,1} \partial_{1}+c_{0,0,0,2} \partial_{2}+c_{0,0,0,3} \partial_{3} .
$$

Since $x_{1} \partial_{1}$ is an idempotent, we can prove that $c_{0,0,0,2}=0, c_{0,0,0,3}=0$. This implies that $D\left(x_{1} \partial_{1}\right)=c_{0,0,0,0}+c_{0,0,0,1} \partial_{1}$. Since $D\left(\partial_{1} * x_{1}^{2} \partial_{1}\right)=$ $2 D\left(x_{1} \partial_{1}\right)$, we are also able to prove that

$$
\begin{aligned}
D\left(x_{1}^{2} \partial_{1}\right) & =2 c_{0,0,0,0} x_{1}+2 c_{0,0,0,1} x_{1} \partial_{1}+\sum_{j, k} t_{0, j, k, 0} x_{2}^{j} x_{3}^{k} \\
& +\sum_{j, k} t_{0, j, k, 1} x_{2}^{j} x_{3}^{k} \partial_{1}+\sum_{j, k} t_{0, j, k, 2} x_{2}^{j} x_{3}^{k} \partial_{2}+\sum_{j, k} t_{0, j, k, 3} x_{2}^{j} x_{3}^{k} \partial_{3}
\end{aligned}
$$

where $t_{0, j, k, 1}, t_{0, j, k, 1}, t_{0, j, k, 2}, t_{0, j, k, 3} \in \mathbb{F}$ for all $j$ and $k$. Since $D\left(x_{1} \partial_{1} *\right.$ $\left.x_{1}^{2} \partial_{1}\right)=2 D\left(x_{1}^{2} \partial_{1}\right)$, we have that $c_{0,0,0,0}=0, t_{0, j, k, 1}=t_{0, j, k, 1}=t_{0, j, k, 2}=$ 
$t_{0, j, k, 3}=0$. This implies that

$$
\begin{aligned}
& D\left(x_{1} \partial_{1}\right)=c_{0,0,0,1} \partial_{1}, \\
& D\left(x_{1}^{2} \partial_{1}\right)=2 c_{0,0,0,1} x \partial_{1}
\end{aligned}
$$

hold. Since $D\left(x_{1} \partial_{1} * x_{1}\right)=D\left(x_{1}\right)$, we also have that $D\left(x_{1}\right)=c_{0,0,0,1}$. By $D\left(\partial_{1} * x_{1}^{3} \partial_{1}\right)=3 D\left(x_{1}^{2} \partial_{1}\right)$, we have that

$$
\begin{aligned}
D\left(x_{1}^{3} \partial_{1}\right) & =3 c_{0,0,0,1} x_{1}^{2} \partial_{1}+\sum_{j, k} s_{0, j, k, 0} x_{2}^{j} x_{3}^{k}+\sum_{j, k} s_{0, j, k, 1} x_{2}^{j} x_{3}^{k} \partial_{1} \\
& +\sum_{j, k} s_{0, j, k, 2} x_{2}^{j} x_{3}^{k} \partial_{2}+\sum_{j, k} s_{0, j, k, 3} x_{2}^{j} x_{3}^{k} \partial_{3},
\end{aligned}
$$

where $s_{0, j, k, 1}, s_{0, j, k, 1}, s_{0, j, k, 2}, s_{0, j, k, 3} \in \mathbb{F}$ for all $j$ and $k$. By $D\left(x_{1} \partial_{1} *\right.$ $\left.x_{1}^{3} \partial_{1}\right)=3 D\left(x_{1}^{3} \partial_{1}\right)$, we have that $D\left(x_{1}^{3} \partial_{1}\right)=3 c_{0,0,0,1} x_{1}^{2} \partial_{1}$. Since $D\left(x_{1}^{2} \partial_{1} *\right.$ $\left.x_{1}^{i-1} \partial_{1}\right)=(i-1) D\left(x_{1}^{i} \partial_{1}\right)$, by induction on $i$ of $x_{1}^{i} \partial_{1}$, we are able to prove that

$$
D\left(x_{1}^{i} \partial_{1}\right)=i c_{0,0,0,1} x_{1}^{i-1} \partial_{1} .
$$

Similarly, we are also able to prove that

$$
\begin{aligned}
& D\left(x_{2}^{j} \partial_{2}\right)=j d_{0,0,0,2} x_{2}^{j-1} \partial_{2}, \\
& D\left(x_{3}^{k} \partial_{3}\right)=j r_{0,0,0,3} x_{3}^{j-1} \partial_{3} .
\end{aligned}
$$

Since $\partial_{u}, 1 \leq u \leq 3$, is in the left annihilator of $x_{1} \partial_{2}$, we can prove that $D\left(x_{1} \partial_{2}\right)=\alpha_{0,0,0,0}+\alpha_{0,0,0,1} \partial_{1}+\alpha_{0,0,0,2} \partial_{2}+\alpha_{0,0,0,3} \partial_{3}$. By $D\left(x_{1} \partial_{1} *\right.$ $\left.x_{1} \partial_{2}\right)=D\left(x_{1} \partial_{2}\right)$, we can also prove that $\alpha_{0,0,0,0}=\alpha_{0,0,0,2}=\alpha_{0,0,0,3}=0$, $\alpha_{0,0,0,1}=c_{0,0,0,1}$. This implies that $D\left(x_{1} \partial_{2}\right)=c_{0,0,0,1} \partial_{2}$. Since $D\left(x_{1}^{2} \partial_{1} *\right.$ $\left.x_{1}^{i-1} \partial_{2}\right)=(i-1) D\left(x_{1}^{i} \partial_{2}\right)$, by induction on $i$ of $x_{1}^{i} \partial_{2}$, we can prove that

$$
D\left(x_{1}^{i} \partial_{2}\right)=i c_{0,0,0,1} x_{1}^{i-1} \partial_{2} .
$$

Similarly, we are able to prove that

$$
\begin{aligned}
& D\left(x_{1}^{i} \partial_{3}\right)=i c_{0,0,0,1} x_{1}^{i-1} \partial_{3}, \\
& D\left(x_{2}^{j} \partial_{u}\right)=j d_{0,0,0,2} x_{2}^{j-1} \partial_{u}, \\
& D\left(x_{3}^{k} \partial_{u}\right)=k r_{0,0,0,3} x_{3}^{k-1} \partial_{u}
\end{aligned}
$$

where $1 \leq u \leq 3$. By $D\left(x_{1}^{i} \partial_{2} * x_{2}^{j+1} \partial_{1}\right)=(j+1) D\left(x_{1}^{i} x_{2}^{j} \partial_{1}\right)$, we have that

$$
D\left(x_{1}^{i} x_{2}^{j} \partial_{1}\right)=i c_{0,0,0,1} x_{1}^{i-1} x_{2}^{j} \partial_{1}+j d_{0,0,0,2} x_{1}^{i} x_{2}^{j-1} \partial_{1} .
$$

Similarly, we are able to prove that

$$
D\left(x_{1}^{i} x_{2}^{j} \partial_{u}\right)=i c_{0,0,0,1} x_{1}^{i-1} x_{2}^{j} \partial_{2}+j d_{0,0,0,2} x_{1}^{i} x_{2}^{j-1} \partial_{u}
$$


where $2 \leq u \leq 3$. Since $D\left(x_{1}^{i} x_{2}^{j} \partial_{3} * x_{3}^{k+1} \partial_{1}\right)=(k+1) D\left(x_{1}^{i} x_{2}^{j} x_{3}^{k} \partial_{1}\right)$, we are also able to prove that

$D\left(x_{1}^{i} x_{2}^{j} x_{3}^{k} \partial_{1}\right)=i c_{0,0,0,1} x_{1}^{i-1} x_{2}^{j} x_{3}^{k} \partial_{1}+j d_{0,0,0,2} x_{1}^{i} x_{2}^{j-1} x_{3}^{k} \partial_{1}+k r_{0,0,0,3} x_{1}^{i} x_{2}^{j} x_{3}^{k-1} \partial_{1}$.

By $D\left(x_{1}^{i} x_{2}^{j} x_{3}^{k} \partial_{1} * x_{1}\right)=D\left(x_{1}^{i} x_{2}^{j} x_{3}^{k}\right)$, we also have that

$$
D\left(x_{1}^{i} x_{2}^{j} x_{3}^{k}\right)=i c_{0,0,0,1} x_{1}^{i-1} x_{2}^{j} x_{3}^{k}+j d_{0,0,0,2} x_{1}^{i} x_{2}^{j-1} x_{3}^{k}+k r_{0,0,0,3} x_{1}^{i} x_{2}^{j} x_{3}^{k-1} .
$$

Similarly, we also have that

$$
\begin{aligned}
D\left(x_{1}^{i} x_{2}^{j} x_{3}^{k} \partial_{u}\right) & =i c_{0,0,0,1} x_{1}^{i-1} x_{2}^{j} x_{3}^{k} \partial_{u}+j d_{0,0,0,2} x_{1}^{i} x_{2}^{j-1} x_{3}^{k} \partial_{u} \\
& +k r_{0,0,0,3} x_{1}^{i} x_{2}^{j} x_{3}^{k-1} \partial_{u}
\end{aligned}
$$

where $2 \leq u \leq 3$. So we have proven the lemma.

Lemma 3.2. For any derivation $D$ of the algebra $W N\left(e^{ \pm x_{1} x_{2} x_{3}}, 0,3\right)_{[1]}$ and for basis elements $x_{1}^{i_{1}} x_{2}^{i_{2}} x_{3}^{i_{3}}, e^{x_{1} x_{2} x_{3}}, e^{-x_{1} x_{2} x_{3}}, x_{1}^{i_{1}} x_{2}^{i_{2}} x_{3}^{i_{3}} \partial_{u}, e^{x_{1} x_{2} x_{3}} \partial_{u}$, $e^{-x_{1} x_{2} x_{3}} \partial_{u}, 1 \leq u \leq 3$, of $W N\left(e^{ \pm x_{1} x_{2} x_{3}}, 0,3\right)_{[1]}$, we have that

$$
\begin{aligned}
& D\left(x_{1}^{i} x_{2}^{j} x_{3}^{k}\right)=0, \\
& D\left(x_{1}^{i} x_{2}^{j} x_{3}^{k} \partial_{u}\right)=0, \\
& D\left(e^{x_{1} x_{2} x_{3}}\right)=c e^{x_{1} x_{2} x_{3}}, \\
& D\left(e^{x_{1} x_{2} x_{3}} \partial_{u}\right)=c e^{x_{1} x_{2} x_{3}} \partial_{u}, \\
& D\left(e^{-x_{1} x_{2} x_{3}}\right)=-c e^{-x_{1} x_{2} x_{3}}, \\
& D\left(e^{-x_{1} x_{2} x_{3}} \partial_{u}\right)=-c e^{-x_{1} x_{2} x_{3}} \partial_{u}
\end{aligned}
$$

hold where $c \in \mathbb{F}$.

Let $D$ be the derivation in the lemma. Since the algebra $W N\left(e^{ \pm x_{1} x_{2} x_{3}}\right.$, $0,3)_{[1]}$ is $\mathbb{Z}$-graded, $D\left(e^{x_{1} x_{2} x_{3}} \partial_{1}\right)$ is the sum of terms in different homogeneous components of $W N\left(e^{ \pm x_{1} x_{2} x_{3}}, 0,3\right)_{[1]}$ in (5). Assume that

$$
\begin{aligned}
D\left(e^{x_{1} x_{2} x_{3}} \partial_{1}\right) & =\sum_{i, j, k \geq 0} a_{i, j, k, 0} e^{p x_{1} x_{2} x_{3}} x_{1}^{i} x_{2}^{j} x_{3}^{k} \\
& +\sum_{i, j, k \geq 0} a_{i, j, k, 1} e^{p x_{1} x_{2} x_{3}} x_{1}^{i} x_{2}^{j} x_{3}^{k} \partial_{1} \\
& +\sum_{i, j, k \geq 0} a_{i, j, k, 2} e^{p x_{1} x_{2} x_{3}} x_{1}^{i} x_{2}^{j} x_{3}^{k} \partial_{2} \\
& +\sum_{i, j, k \geq 0} a_{i, j, k, 3} e^{p x_{1} x_{2} x_{3}} x_{1}^{i} x_{2}^{j} x_{3}^{k} \partial_{3}
\end{aligned}
$$


with appropriate coefficients. We have that

$$
\begin{aligned}
D\left(\partial_{1} * e^{x_{1} x_{2} x_{3}} \partial_{1}\right) & =D\left(e^{x_{1} x_{2} x_{3}} x_{2} x_{3} \partial_{1}\right) \\
& =\sum_{i, j, k \geq 0} p a_{i, j, k, 0} e^{p x_{1} x_{2} x_{3}} x_{1}^{i} x_{2}^{j+1} x_{3}^{k+1} \\
& +\sum_{j, k \geq 0, i \geq 1} i a_{i, j, k, 0} e^{p x_{1} x_{2} x_{3}} x_{1}^{i-1} x_{2}^{j} x_{3}^{k} \\
& +\sum_{i, j, k \geq 0} p a_{i, j, k, 1} e^{p x_{1} x_{2} x_{3}} x_{1}^{i} x_{2}^{j+1} x_{3}^{k+1} \partial_{1} \\
& +\sum_{j, k \geq 0, i \geq 1} i a_{i, j, k, 1} e^{p x_{1} x_{2} x_{3}} x_{1}^{i-1} x_{2}^{j} x_{3}^{k} \partial_{1} \\
& +\sum_{i, j, k \geq 0} p a_{i, j, k, 2} e^{p x_{1} x_{2} x_{3}} x_{1}^{i} x_{2}^{j+1} x_{3}^{k+1} \partial_{2} \\
& +\sum_{j, k \geq 0, i \geq 1} i a_{i, j, k, 2} e^{p x_{1} x_{2} x_{3}} x_{1}^{i-1} x_{2}^{j} x_{3}^{k} \partial_{2} \\
& +\sum_{i, j, k \geq 0} p a_{i, j, k, 3} e^{p x_{1} x_{2} x_{3}} x_{1}^{i} x_{2}^{j+1} x_{3}^{k+1} \partial_{3} \\
& +\sum_{j, k \geq 0, i \geq 1} i a_{i, j, k, 3} e^{p x_{1} x_{2} x_{3}} x_{1}^{i-1} x_{2}^{j} x_{3}^{k} \partial_{3}
\end{aligned}
$$

$(8)$

and

$$
\begin{aligned}
D\left(e^{x_{1} x_{2} x_{3}} \partial_{1} * x_{1} x_{2} x_{3} \partial_{1}\right) & =D\left(e^{x_{1} x_{2} x_{3}} x_{2} x_{3} \partial_{1}\right) \\
& =\sum_{i, j, k \geq 0} a_{i, j, k, 0} e^{p x_{1} x_{2} x_{3}} x_{1}^{i+1} x_{2}^{j+1} x_{3}^{k+1} \\
& +\sum_{i, j, k \geq 0} a_{i, j, k, 1} e^{p x_{1} x_{2} x_{3}} x_{1}^{i} x_{2}^{j+1} x_{3}^{k+1} \partial_{1} \\
& +\sum_{i, j, k \geq 0} a_{i, j, k, 2} e^{p x_{1} x_{2} x_{3}} x_{1}^{i+1} x_{2}^{j} x_{3}^{k+1} \partial_{1} \\
& +\sum_{i, j, k \geq 0} a_{i, j, k, 3} e^{p x_{1} x_{2} x_{3}} x_{1}^{i+1} x_{2}^{j+1} x_{3}^{k} \partial_{1} \\
& +d_{0,0,0,2} e^{x_{1} x_{2} x_{3}} x_{3} \partial_{1}+r_{0,0,0,3} e^{x_{1} x_{2} x_{3}} x_{2} \partial_{1} .
\end{aligned}
$$

By comparing (8) and (9), we have that $p=1, a_{i, j, k, 0}=a_{i, j, k, 2}=$ $a_{i, j, k, 3}=0, i, j, k \geq 0, a_{i, j, k, 1}=0, i \geq 1$, and $d_{0,0,0,2}=r_{0,0,0,3}=0$. This 
implies that

$$
D\left(e^{x_{1} x_{2} x_{3}} \partial_{1}\right)=\sum_{j, k \geq 0} a_{0, j, k, 1} e^{x_{1} x_{2} x_{3}} x_{2}^{j} x_{3}^{k} \partial_{1} .
$$

Since

$$
\begin{aligned}
D\left(\partial_{2} * e^{x_{1} x_{2} x_{3}} \partial_{1}\right) & =D\left(e^{x_{1} x_{2} x_{3}} x_{1} x_{3} \partial_{1}\right) \\
& =\sum_{j, k \geq 0} a_{0, j, k, 1} e^{x_{1} x_{2} x_{3}} x_{1} x_{2}^{j} x_{3}^{k+1} \partial_{1} \\
& +\sum_{j \geq 1, k \geq 0} j a_{0, j, k, 1} e^{x_{1} x_{2} x_{3}} x_{2}^{j-1} x_{3}^{k} \partial_{1}
\end{aligned}
$$

and

$$
\begin{aligned}
D\left(e^{x_{1} x_{2} x_{3}} \partial_{1} * x_{1}^{2} x_{3} \partial_{1}\right) & =2 D\left(e^{x_{1} x_{2} x_{3}} x_{1} x_{3} \partial_{1}\right) \\
& =2 \sum_{j, k \geq 0} a_{0, j, k, 1} e^{x_{1} x_{2} x_{3}} x_{1} x_{2}^{j} x_{3}^{k+1} \partial_{1} \\
& +2 c_{0,0,0,1} e^{x_{1} x_{2} x_{3}} x_{3} \partial_{1},
\end{aligned}
$$

we have that $a_{0, j, k, 1}=0, j \geq 1$ and $c_{0,0,0,1}=0$. This implies that

$$
D\left(e^{x_{1} x_{2} x_{3}} \partial_{1}\right)=\sum_{k \geq 0} a_{0,0, k, 1} e^{x_{1} x_{2} x_{3}} x_{3}^{k} \partial_{1} .
$$

Since

$$
\begin{aligned}
D\left(\partial_{3} * e^{x_{1} x_{2} x_{3}} \partial_{1}\right) & =D\left(e^{x_{1} x_{2} x_{3}} x_{1} x_{2} \partial_{1}\right) \\
& =\sum_{k \geq 0} a_{0,0, k, 1} e^{x_{1} x_{2} x_{3}} x_{1} x_{2} x_{3}^{k} \partial_{1} \\
& +\sum_{k \geq 1} k a_{0,0, k, 1} e^{x_{1} x_{2} x_{3}} x_{3}^{k-1} \partial_{1}
\end{aligned}
$$

and

$$
\begin{aligned}
D\left(e^{x_{1} x_{2} x_{3}} \partial_{1} * x_{1}^{2} x_{2} \partial_{1}\right) & =2 D\left(e^{x_{1} x_{2} x_{3}} x_{1} x_{2} \partial_{1}\right) \\
& =2 \sum_{k \geq 0} a_{0,0, k, 1} e^{x_{1} x_{2} x_{3}} x_{1} x_{2} x_{3}^{k} \partial_{1},
\end{aligned}
$$

we have that $a_{0,0, k, 1}=0, k \geq 1$. This implies that

$$
D\left(e^{x_{1} x_{2} x_{3}} \partial_{1}\right)=a_{0,0,0,1} e^{x_{1} x_{2} x_{3}} \partial_{1}
$$


and we also have

$$
\begin{aligned}
& D\left(x_{1}^{i} \partial_{u}\right)=0, \\
& D\left(x_{2}^{j} \partial_{u}\right)=0, \\
& D\left(x_{3}^{k} \partial_{u}\right)=0, \\
& D\left(x_{1}^{i} x_{2}^{j} x_{3}^{k}\right)=0, \\
& D\left(x_{1}^{i} x_{2}^{j} x_{3}^{k} \partial_{u}\right)=0
\end{aligned}
$$

where $2 \leq u \leq 3$. By (12) and $D\left(e^{x_{1} x_{2} x_{3}} \partial_{1} * x_{1}\right)=D\left(e^{x_{1} x_{2} x_{3}}\right)$, we also have that $D\left(e^{x_{1} x_{2} x_{3}}\right)=a_{0,0,0,1} e^{x_{1} x_{2} x_{3}}$. By $D\left(e^{x_{1} x_{2} x_{3}} \partial_{1} * x_{1} \partial_{2}\right)=$ $D\left(e^{x_{1} x_{2} x_{3}} \partial_{2}\right)$ and $D\left(e^{x_{1} x_{2} x_{3}} \partial_{1} * x_{1} \partial_{3}\right)=D\left(e^{x_{1} x_{2} x_{3}} \partial_{3}\right)$, we can prove that

$$
\begin{aligned}
& D\left(e^{x_{1} x_{2} x_{3}} \partial_{2}\right)=a_{0,0,0,1} e^{x_{1} x_{2} x_{3}} \partial_{2}, \\
& D\left(e^{x_{1} x_{2} x_{3}} \partial_{3}\right)=a_{0,0,0,1} e^{x_{1} x_{2} x_{3}} \partial_{3} .
\end{aligned}
$$

Since $D\left(e^{x_{1} x_{2} x_{3}} \partial_{1} * e^{-x_{1} x_{2} x_{3}} \partial_{1}\right)=0$, we can prove that

$$
\begin{aligned}
D\left(e^{-x_{1} x_{2} x_{3}} \partial_{1}\right) & =-a_{0,0,0,1} e^{-x_{1} x_{2} x_{3}} \partial_{1}+\sum_{1 \leq u \leq 3, j, k \geq 0} \beta_{0, j, k, 0} x_{2}^{j} x_{3}^{k} \\
& +\sum_{j, k \geq 0} \beta_{0, j, k, 1} x_{2}^{j} x_{3}^{k} \partial_{1}+\sum_{j, k \geq 0} \beta_{0, j, k, 2} x_{2}^{j} x_{3}^{k} \partial_{2} \\
& +\sum_{j, k \geq 0} \beta_{0, j, k, 3} x_{2}^{j} x_{3}^{k} \partial_{3} .
\end{aligned}
$$

By $D\left(e^{-x_{1} x_{2} x_{3}} \partial_{1} * x_{1} \partial_{1}\right)=D\left(e^{-x_{1} x_{2} x_{3}} \partial_{1}\right)$, we can also prove that

$$
D\left(e^{-x_{1} x_{2} x_{3}} \partial_{1}\right)=-a_{0,0,0,1} e^{-x_{1} x_{2} x_{3}} \partial_{1} .
$$

By $D\left(e^{-x_{1} x_{2} x_{3}} \partial_{1} * x_{1}\right)=D\left(e^{-x_{1} x_{2} x_{3}}\right)$, we also have that

$$
D\left(e^{-x_{1} x_{2} x_{3}}\right)=-a_{0,0,0,1} e^{-x_{1} x_{2} x_{3}} .
$$

Similarly, we can prove that

$$
\begin{aligned}
& D\left(e^{-x_{1} x_{2} x_{3}} \partial_{2}\right)=-a_{0,0,0,1} e^{-x_{1} x_{2} x_{3}} \partial_{2}, \\
& D\left(e^{-x_{1} x_{2} x_{3}} \partial_{3}\right)=-a_{0,0,0,1} \cdot e^{-x_{1} x_{2} x_{3}} \partial_{3} .
\end{aligned}
$$

So we have proven the lemma.

Theorem 3.1. For any derivation $D$ of the algebra $W N\left(e^{ \pm x_{1} x_{2} x_{3}}, 0,3\right)_{[1]}$ and for basis elements 
$e^{p x_{1} x_{2} x_{3}} x_{1}^{i} x_{2}^{j} x_{3}^{k}$ and $e^{p x_{1} x_{2} x_{3}} x_{1}^{i} x_{2}^{j} x_{3}^{k} \partial_{u}, 1 \leq u \leq 3$, of $W N\left(e^{ \pm x_{1} x_{2} x_{3}}, 0,3\right)_{[1]}$, we have that

$$
\begin{aligned}
& D\left(e^{p x_{1} x_{2} x_{3}} x_{1}^{i} x_{2}^{j} x_{3}^{k}\right)=p c e^{p x_{1} x_{2} x_{3}} x_{1}^{i} x_{2}^{j} x_{3}^{k}, \\
& D\left(e^{p x_{1} x_{2} x_{3}} x_{1}^{i} x_{2}^{j} x_{3}^{k} \partial_{u}\right)=p c e^{p x_{1} x_{2} x_{3}} x_{1}^{i} x_{2}^{j} x_{3}^{k} \partial_{u}
\end{aligned}
$$

hold where $1 \leq u \leq 3, p \in \mathbb{Z}$, and $c \in \mathbb{F}$.

Proof. Let $D$ be the derivation in the lemma. By $D\left(e^{x_{1} x_{2} x_{3}} \partial_{1} *\right.$ $\left.x_{1}^{i+1} \partial_{u}\right)=(i+1) D\left(e^{x_{1} x_{2} x_{3}} x_{1}^{i} \partial_{u}\right)$, we are able to prove that $D\left(e^{x_{1} x_{2} x_{3}} x_{1}^{i} \partial_{u}\right)$ $=a_{0,0,0,1} e^{x_{1} x_{2} x_{3}} x_{1}^{i} \partial_{u}$ for $1 \leq u \leq 3$, with appropriate coefficients. By $D\left(e^{x_{1} x_{2} x_{3}} \partial_{2} * e^{x_{1} x_{2} x_{3}} \partial_{u}\right)=\bar{D}\left(e^{2 x_{1} x_{2} x_{3}} x_{1} x_{3} \partial_{u}\right)$, we are also able to prove that

$$
D\left(e^{2 x_{1} x_{2} x_{3}} x_{1} x_{3} \partial_{u}\right)=2 a_{0,0,0,1} e^{2 x_{1} x_{2} x_{3}} x_{1} x_{3} \partial_{u} .
$$

Since $D\left(e^{x_{1} x_{2} x_{3}} x_{1} \partial_{2} * e^{x_{1} x_{2} x_{3}} x_{1}^{i-2} \partial_{u}\right)=D\left(e^{2 x_{1} x_{2} x_{3}} x_{1}^{i} x_{3} \partial_{u}\right)$, we also have that

$$
D\left(e^{2 x_{1} x_{2} x_{3}} x_{1}^{i} x_{3} \partial_{u}\right)=2 a_{0,0,0,1} e^{2 x_{1} x_{2} x_{3}} x_{1}^{i} x_{3} \partial_{u} .
$$

By $D\left(e^{x_{1} x_{2} x_{3}} x_{1} x_{3} \partial_{2} * e^{x_{1} x_{2} x_{3}} x_{1}^{i-2} \partial_{u}\right)=D\left(e^{2 x_{1} x_{2} x_{3}} x_{1}^{i} x_{3}^{2} \partial_{u}\right)$, we prove that

$$
D\left(e^{2 x_{1} x_{2} x_{3}} x_{1}^{i} x_{3}^{2} \partial_{u}\right)=2 a_{0,0,0,1} e^{2 x_{1} x_{2} x_{3}} x_{1}^{i} x_{3}^{2} \partial_{u},
$$

and by $D\left(e^{2 x_{1} x_{2} x_{3}} x_{1}^{i} x_{3}^{2} \partial_{3} * x_{3}^{k-1} \partial_{u}\right)=(k-1) D\left(e^{2 x_{1} x_{2} x_{3}} x_{1}^{i} x_{3}^{k} \partial_{u}\right)$, we also prove that

$$
D\left(e^{2 x_{1} x_{2} x_{3}} x_{1}^{i} x_{3}^{k} \partial_{u}\right)=2 a_{0,0,0,1} e^{2 x_{1} x_{2} x_{3}} x_{1}^{i} x_{3}^{k} \partial_{u} .
$$

By $D\left(e^{2 x_{1} x_{2} x_{3}} x_{1}^{i} x_{3}^{k} \partial_{3} * x_{2}^{j} x_{3} \partial_{u}\right)=D\left(e^{2 x_{1} x_{2} x_{3}} x_{1}^{i} x_{2}^{j} x_{3}^{k} \partial_{u}\right)$, we have that

$$
D\left(e^{2 x_{1} x_{2} x_{3}} x_{1}^{i} x_{2}^{j} x_{3}^{k} \partial_{u}\right)=2 a_{0,0,0,1} e^{2 x_{1} x_{2} x_{3}} x_{1}^{i} x_{2}^{j} x_{3}^{k} \partial_{u},
$$

and by $D\left(e^{2 x_{1} x_{2} x_{3}} x_{1}^{i} x_{2}^{j-1} x_{3}^{k-1} \partial_{1} * e^{x_{1} x_{2} x_{3}} \partial_{u}\right)=D\left(e^{3 x_{1} x_{2} x_{3}} x_{1}^{i} x_{2}^{j} x_{3}^{k} \partial_{u}\right)$, we also have that

$$
D\left(e^{3 x_{1} x_{2} x_{3}} x_{1}^{i} x_{2}^{j} x_{3}^{k} \partial_{u}\right)=3 a_{0,0,0,1} e^{3 x_{1} x_{2} x_{3}} x_{1}^{i} x_{2}^{j} x_{3}^{k} \partial_{u} .
$$

By induction on $p \in \mathbb{Z}$ of $e^{p x_{1} x_{2} x_{3}} x_{1}^{i} x_{2}^{j} x_{3}^{k} \partial_{u}$ and $D\left(e^{(p-1) x_{1} x_{2} x_{3}} x_{1}^{i} x_{2}^{j-1} x_{3}^{k-1}\right.$ $\left.\partial_{1} * e^{x_{1} x_{2} x_{3}} \partial_{u}\right)=D\left(e^{p x_{1} x_{2} x_{3}} x_{1}^{i} x_{2}^{j} x_{3}^{k} \partial_{u}\right)$, we are able to prove that

$$
D\left(e^{p x_{1} x_{2} x_{3}} x_{1}^{i} x_{2}^{j} x_{3}^{k} \partial_{u}\right)=p a_{0,0,0,1} e^{p x_{1} x_{2} x_{3}} x_{1}^{i} x_{2}^{j} x_{3}^{k} \partial_{u} .
$$

By putting $c=a_{0,0,0,1}$, we have that

$$
D\left(e^{p x_{1} x_{2} x_{3}} x_{1}^{i} x_{2}^{j} x_{3}^{k} \partial_{u}\right)=p c e^{p x_{1} x_{2} x_{3}} x_{1}^{i} x_{2}^{j} x_{3}^{k} \partial_{u} .
$$

By $D\left(e^{p x_{1} x_{2} x_{3}} x_{1}^{i} x_{2}^{j} x_{3}^{k} \partial_{1} * x_{1}\right)=D\left(e^{p x_{1} x_{2} x_{3}} x_{1}^{i} x_{2}^{j} x_{3}^{k}\right)$, we also have that

$$
D\left(e^{p x_{1} x_{2} x_{3}} x_{1}^{i} x_{2}^{j} x_{3}^{k}\right)=p c e^{p x_{1} x_{2} x_{3}} x_{1}^{i} x_{2}^{j} x_{3}^{k} .
$$


Therefore we have proven the lemma.

Theorem 3.2. For any $D \in \operatorname{Der}_{n o n}\left(W N\left(e^{ \pm x_{1} x_{2} x_{3}}, 0,3\right)_{[1]}\right), D$ is the linear sum of the derivations $D_{c}$ as shown in Note 1 where $c \in \mathbb{F}$. The additive group $D \in \operatorname{Der}_{n o n}\left(W N\left(e^{ \pm x_{1} x_{2} x_{3}}, 0,3\right)_{[1]}\right)$ is isomorphic to the additive group $\mathbb{F}$. Every derivation of the algebra $W N\left(e^{ \pm x_{1}^{r}}, 0,3\right)_{[1]}$ is outer.

Proof. The proofs of the theorem are straightforward by Lemma 3.2, Theorem 3.1, and the fact that the derivation of Note 1 cannot be inner. This completes the proof of the theorem.

Corollary 3.1. The dimension of $\operatorname{Der}_{n o n}\left(W N\left(e^{ \pm x_{1} x_{2} x_{3}}, 0,3\right)_{[1]}\right)$ of the algebra $W N\left(e^{ \pm x_{1} x_{2} x_{3}}, 0,3\right)[1]$ is one. For any derivation $D$ of $D e r_{n o n}$ $\left(W N\left(e^{ \pm x_{1} x_{2} x_{3}}, 0,3\right)_{[1]}\right), D\left(N_{0}^{\prime}\right)=0$ holds where $N_{0}^{\prime}$ is the zero-homogeneous component of $W N\left(e^{ \pm x_{1} x_{2} x_{3}}, 0,3\right)_{[1]}$ in (5) (see [8] and [9]).

Proof. The proofs of the corollary are straightforward by Lemma 3.2 and Note 1.

Proposition 3.1. If $A$ is not a purely outer algebra, then algebra $A$ and $W N\left(e^{ \pm x_{1} x_{2} x_{3}}, 0,3\right)_{[1]}$ are not isomorphic.

Proof. The proof of the proposition is straightforward by Theorem 3.2 .

Remarks. By Theorem 3.2, there is a class $\mathfrak{P}$ of purely outer algebras, i.e., for any $A \in \mathfrak{P}$ and for any $D \in \operatorname{Der}(A), D$ is outer.

\section{References}

[1] Mohammad H. Ahmadi, Ki-Bong Nam, and Jonathan Pakianathan, Lie admissible non-associative algebras, Algebra Colloquium, Vol. 12, No. 1, World Scientific, (March) 2005, 113-120.

[2] Seul Hee Choi and Ki-Bong Nam, The Derivation of a Restricted Weyl Type NonAssociative Algebra, Vol. 28, No. 3, Hadronic Journal, 2005, 287-295.

[3] Seul Hee Choi, An algebra with right identities and its antisymmetrized algebra, Honam Mathematical Journal, Vol. 29, No. 2, 2007, 213-222.

[4] Seul Hee Choi, New algebras using additive abelian groups I, Honam Mathematical Journal, Vol. 31, No. 3, 2009, 407-419. 
[5] Seul Hee Choi and Ki-Bong Nam, "Weyl type non-associative algebra using additive groups I," Algebra Colloquium, Volume 14 (2007), 479-488, Number 3, 2007.

[6] Seul Hee Choi and Ki-Bong Nam, "Derivations of a restricted Weyl Type Algebra I", Rocky Mountain Math. Journals, Volume 37, Number 6, 2007, 67-84.

[7] Seul Hee Choi, Jongwoo Lee, and Ki-Bong Nam, "Derivations of a restricted Weyl type algebra containing the polynomial ring", Communication in Algebra, Volume 36, Issue 9 September 2008, 3435 - 3446.

[8] I. N. Herstein, Noncommutative Rings, Carus Mathematical Monographs, Mathematical association of America, 100-101.

[9] J. E. Humphreys, Introduction to Lie Algebras and Representation Theory, Springer-Verlag, New York, 1987, 7-21.

[10] T. Ikeda, N. Kawamoto and Ki-Bong Nam, A class of simple subalgebras of Generalized $W$ algebras, Proceedings of the International Conference in 1998 at Pusan (Eds. A. C. Kim), Walter de Gruyter Gmbh Co. KG, 2000, 189-202.

[11] V. G. Kac, DeX-X-SCRIPTion of Filtered Lie Algebra with which Graded Lie algebras of Cartan type are Associated, Izv. Akad. Nauk SSSR, Ser. Mat. Tom, 38, 1974, 832-834.

[12] Naoki Kawamoto, Atsushi Mitsukawa, Ki-Bong Nam, and Moon-Ok Wang, The automorphisms of generalized Witt type Lie algebras, Journal of Lie Theory, 13 Vol(2), Heldermann Verlag, 2003, 571-576.

[13] Jongwoo Lee and Ki-bong Nam, "Non-Associative Algebras containing the Matrix Ring", Linear Algebra and its Applications Volume 429, Issue 1, 1 July 2008, Pages 72-78.

[14] Ki-Bong Nam, Generalized $W$ and H Type Lie Algebras, Algebra Colloquium 6:3, (1999), 329-340.

[15] Ki-Bong Nam, On Some Non-Associative Algebras Using Additive Groups, Southeast Asian Bulletin of Mathematics, Vol. 27, Springer Verlag, 2003, 493-500.

[16] Ki-Bong Nam and Moon-Ok Wang, Notes on Some Non-Associative Algebras, Journal of Applied Algebra and Discrete Structured, Vol 1, No. 3, 159-164.

[17] D. P. Passman, Simple Lie algebras of Witt type, J. Algebra 206 (1998).

[18] R. D. Schafer, Introduction to nonassociative algebras, Dover, 1995, 128-138. 
Dept. of Mathematics,

Jeonju University,

Chon-ju 560-759, Korea, 